\title{
Design of Broadband RoF PON for the Last Mile
}

\author{
Ayoob Alateeq, Khaled Alatawi, Fahad Almasoudi, Mohammad A. Matin \\ Department Electrical and Computer Engineering, University of Denver, Denver, USA \\ Email: Ayoob.alateeq@du.edu
}

Received August 24, 2012; revised September 22, 2012; accepted October 25, 2012

\begin{abstract}
An increase in the demand of broadband service has encouraged research and study to find a solution to offer an adequate amount of service. Living in this digital world with downloading video, voice or data leads us occasionally to have a shortage of bandwidth in the provided data. One of the solutions to cover the huge expected demand in the future is improving the communication systems by adding optical passive components to the Radio over Fiber (RoF) system. This work is mainly to increase the bandwidth that allows the small and single consumer at the last mile. We have shown that by adding the 40-GHz mm-wave to the system, Bit-Error-Rate (BER) has increased while Q-factor has decreased.
\end{abstract}

Keywords: WDM-PON; MM-Wave Radio; RoF; Q-Factor; BER

\section{Introduction}

In the modern communication systems, optical fiber is used to promote the efficiency in the transmitting and the receiving signals. This development in the communication system will help to supply enough broadband service to the last point of each individual consumer. Providing broadband service to each single end would not be an easy task to the service providers. Not only does optical fiber have immunity to the electromagnetic field, it also offers a high bandwidth that might be needed at the end of a single terminal. Distributing services to each single end will be costly. However, by using the wavelength division multiplexer (WDM), which works to transmit one signal with many wavelengths, one fiber, is needed to connect the wavelength division multiplexing to the demultiplexing at the end of the transmitter side and the beginning of the receiver side. Fiber to terminal $x$ technology (FTTx) has shown its ability to increase the bandwidth at the last mile terminals such as small businesses and housing customers. The passive optical network (PON), which consists of, the optical line terminal (OLN), the single mode fiber optic (SM), and the optical network terminal (ONT), is simply a (FTTx) technology. WDM PON systems can also offer symmetric wavelengths, both downstream (from OLN to ONT) and upstream (from ONT to OLN) [1]. Moreover, for the wireless broadband technology $\mathrm{mm}$-wave radio shows its efficiency in supporting the wireless connections. In this paper, we have found the function of WDM-PON systems which requires having all passive optical components and studying the system after adding the optical fiber to the last mile terminal [2]. We also have added mm-wave to the carrier signal in order to take the advantage of mm-wave.

The demand for a high-speed data rate has increased over the last few years. The traditional networks that use coaxial cable and wireless communication have become insufficient to provide high data and large bandwidth. To overcome this problem, an optical fiber must be used because it can provide large bandwidth and a high data transfer rate. The use of the passive optical network (PON) is the most efficient and economic way to solve this problem because of its characteristics. The passive optical network has many advantages. For instance, it does not use active devices such as optical amplifiers, repeaters and active splitters especially between the central offices and the base stations. Therefore it consumes less power, less space, and less complexity. Because of this it can be expanded cheaply as compared to the active network [3].

\section{System Design}

The most dominant use of the passive optical network is to provide high speed, large bandwidth, and a high data rate to the end user at the last mile. The demand for using the Internet service for gaming, video calling, and highdefinition television has been increased by the end users [1]. Therefore, this will require large bandwidth and a high data rate. The main goal of this project is to provide high-speed Internet services to the last mile by using fiber to the home, which is known as FTTH. In this study we used the broadband passive optical network, which is 
known as BPON to implement the FTTH network and adding $\mathrm{mm}$-wave to the carrier and finding the effects that $\mathrm{mm}$-wave apply to the output signal.

In this paper we design and analysis a broadband passive optical network (BPON-RoF) to achieve a highspeed data rate and large bandwidth. We have built our system in two different designs one is without a mmwave and the second one is with a mm-wave. OptiSystem software version 10 was used to simulate those two designs. Figure 1 shows the basic Architecture of WDM-PON network. Figure 2 represents our whole model after adding the $40-\mathrm{GHz}$ mm-wave. We use two transmitters that have been used to generate two different wavelengths of 1510 and $1530 \mathrm{~nm}$, respectively. The two wavelengths have been combined using a WDM multiplexer. After that the signal has been inserted in the bidirectional single mode optical fiber. After using the bidirectional SM optical fiber a splitter was used as in Figure 3. The splitter block contains the WDM demultiplexer to separate the two wavelengths in the downstream. For the upstream the splitter block contains WDM in order to combine the wavelengths that will be

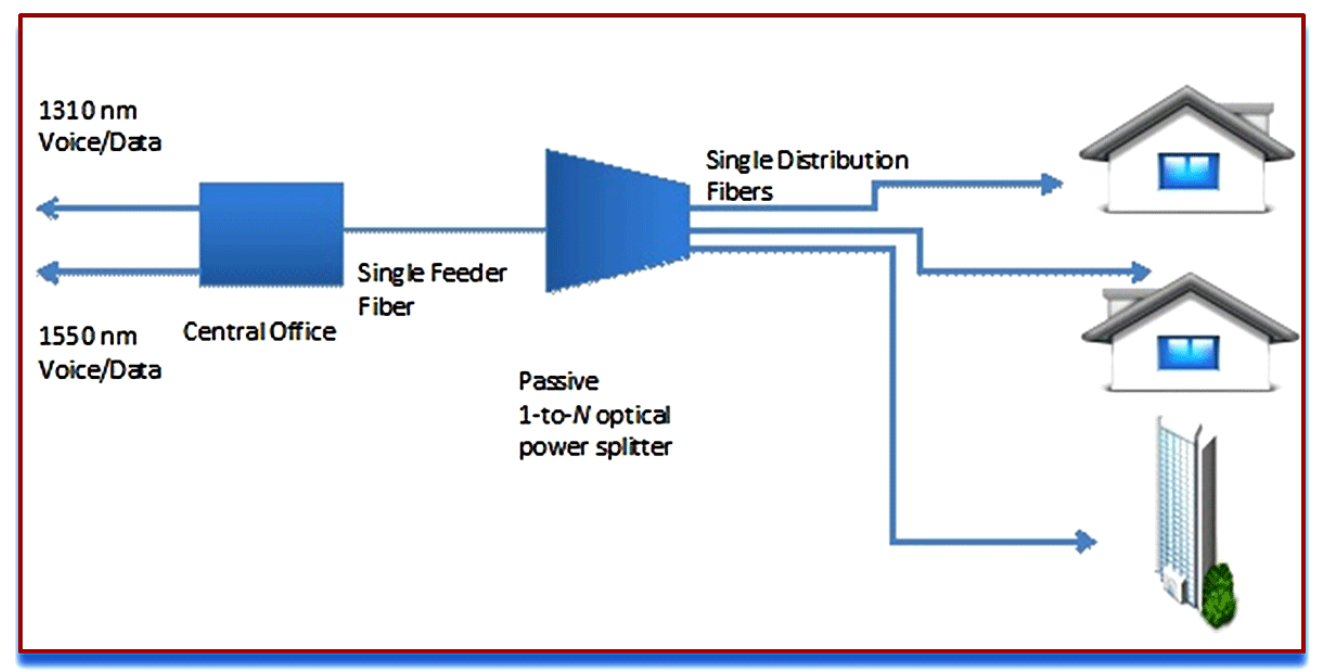

Figure 1. Basic architecture of WDM-PON network.

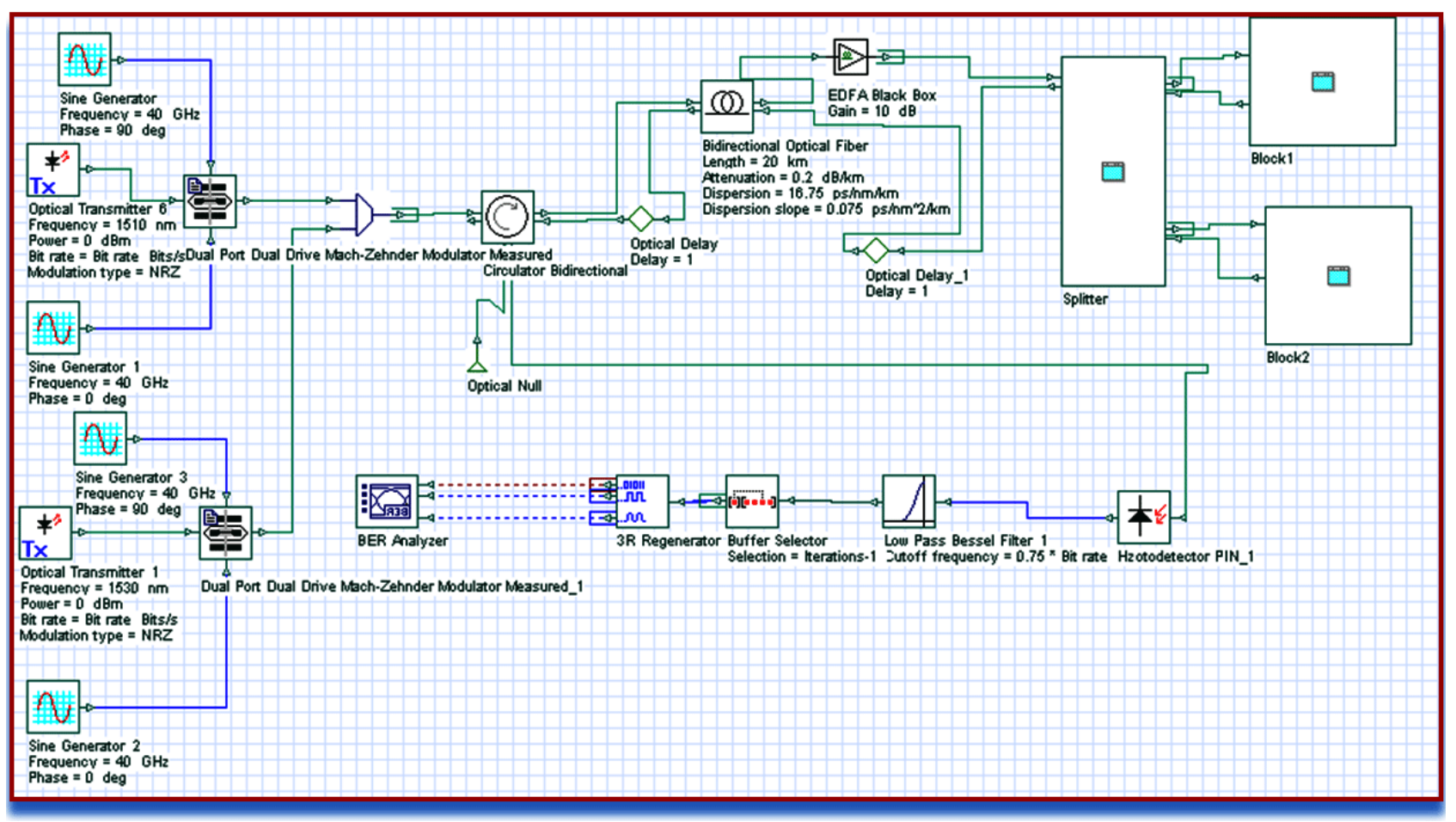

Figure 2. System model. 
sent by the end users. For the downstream the two wavelengths that were demultiplexed will be transmitted to the two optical network terminals (ONTs). Each ONT contains an optical splitter and to optical network units (ONUs). The signal that comes from the splitter block will be inserted into the splitter through $3 \mathrm{Km}$ bidirectional optical fiber, after dividing the signal each output port from the splitter will deliver a signal to an ONU through $50 \mathrm{~m}$ bidirectional optical as shown in Figure 4. Each ONU consists of a photodetector with a low pass filter for the downstream and a transmitter for the upstream signals as shown in Figure 5. At each of the

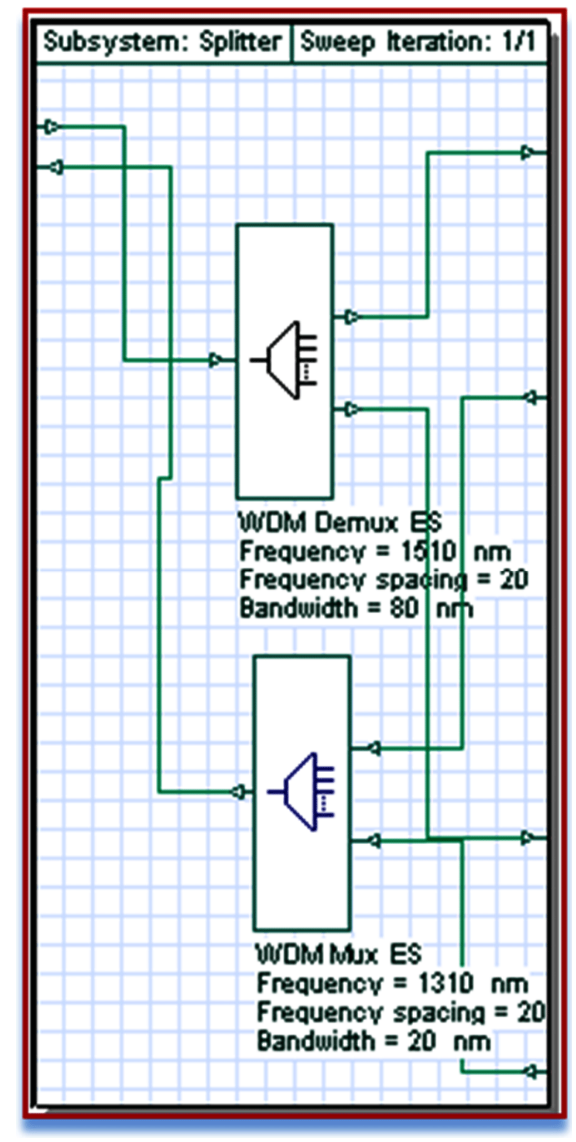

Figure 3. Splitter Circuit with WDM Mux and Dmux.
ONUs the transmitter's wavelength is $1310 \mathrm{~nm}$. Since we have four transmitters with the same wavelength that may transmit their information at the same time, we take the advantage of time division multiplexing access (TDMA) to design the upstream system. TDMA has been designed by using two dynamic y select that will pass the signal upstream in a determined period of time and will set the rest of the signals to zero Figure 5. The receiver end of the upstream link consists of a buffer selector to select the desired signal. A photodetector with low pass filter is connected to the buffer selector. By using dualarm MZM in the input side, we have inserted $40-\mathrm{GHz}$ mm-wave signal based on optical carrier suppression (OCS) to the input signal 1510 and $1530 \mathrm{~nm}$ for the downstream link; however, for the upstream link out of MZM was connected to the input of the selectors [3-5].

\section{Design Studying Parameters}

After designing the system, three parameters were tested to study the performance of the network and the quality of the signal. These parameters are the Q-factor, the bit error rate and the eye diagram. The Q-factor is the quality factor; a higher Q-factor indicates a higher signal quality. On the other hand, the bit error rate (BER) is the ratio between the number of the bits with errors and the total number of bits received and it helps to identify the qua- lity of the optical connection. The eye diagram is one of the important methods to study the system. The eye opening can indicate the noise in the signal and how it differentiates the logic 0 from logic 1 . The eye width can indicate the jitter effect and the rising or falling edge can indicate the distortion of the signal path [6,7].

\section{Results}

After designing this PON, a number of parameters have to be considered such as bit rate, sequence length, samples per bit and the total samples. In the case we have studied this system is at $2.5 \mathrm{G} / \mathrm{s}$ as a bit rate, the sequence length is 128 bits, samples per bit are 64 and the total number of samples is 8192 .

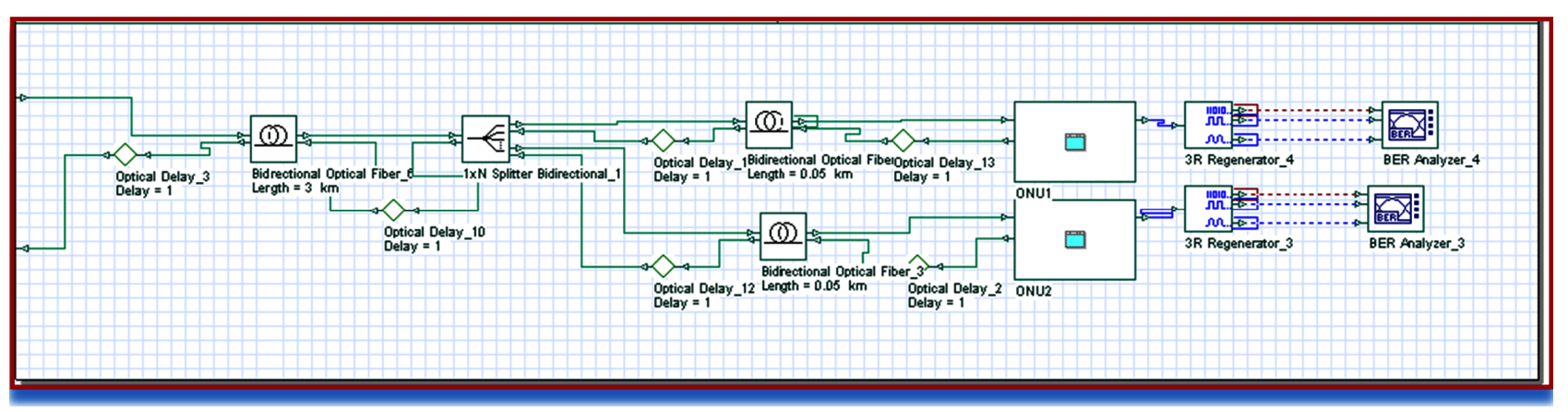

Figure 4. Passive optical network for one block. 


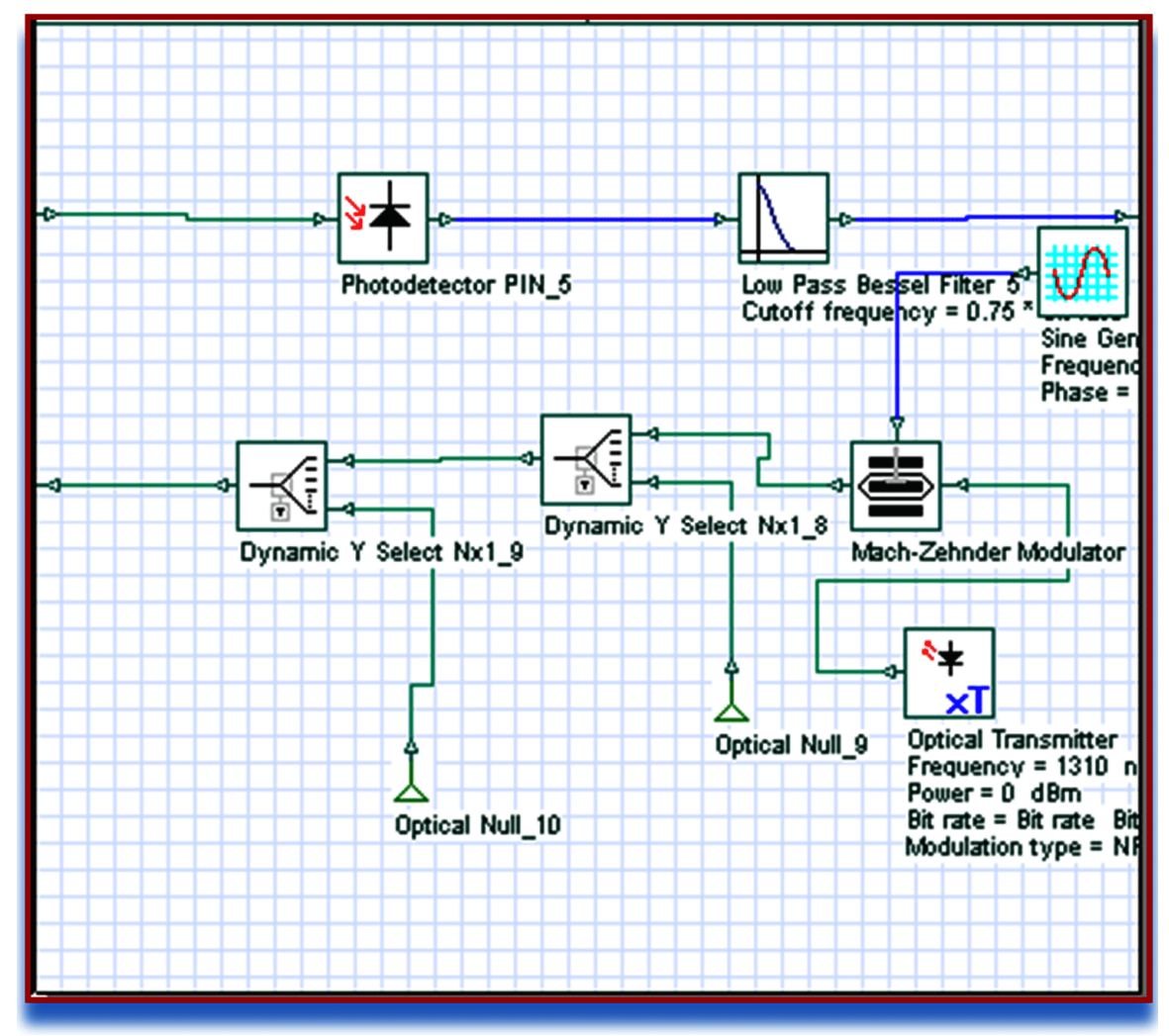

Figure 5. Optical network unit (ONU).

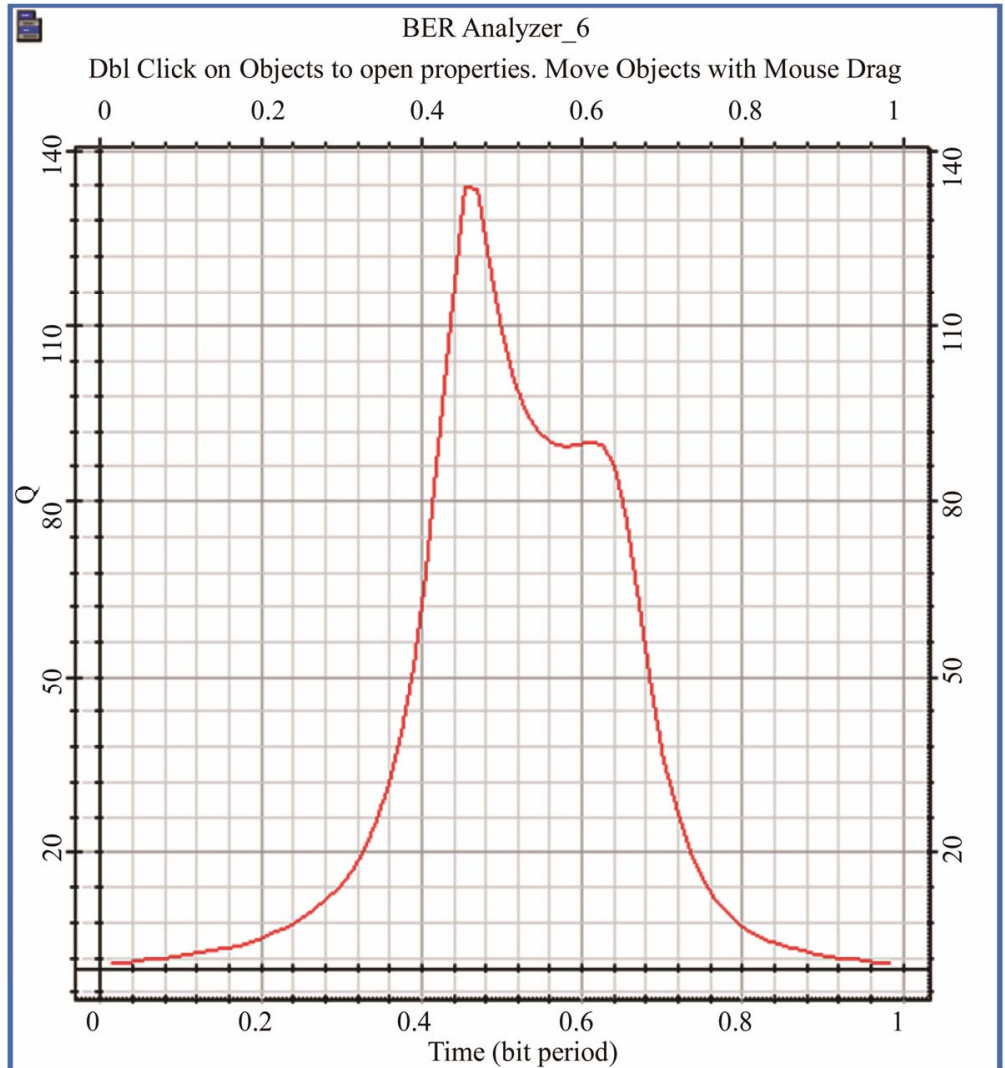

(a) 


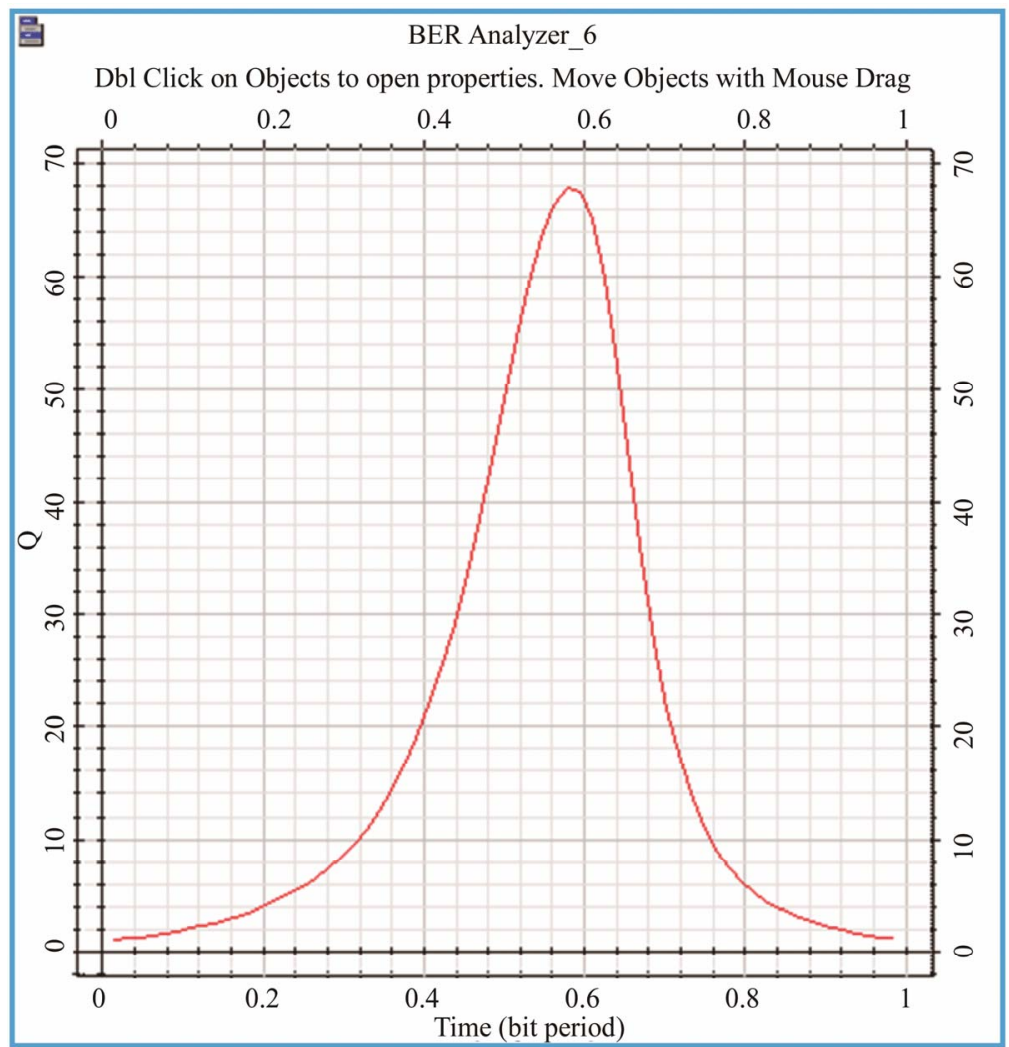

(b)

Figure 6. Q-factor vs. time for downstream signal with (b) and without (a) 40-GHz mm-wave.

\subsection{Downstream Design Analysis}

For the downstream signal with and without adding $\mathrm{mm}$-wave to the system, Table 1 shows the important output of BER, Q-factor and eye diagram which are at one single user. Because of having almost identical outputs for each one of the four users, we have analyzed the quality of the signals at only one single user. From Figure 6(a), the maximum $\mathrm{Q}$-factor is equal to 133.819; however, after adding $40-\mathrm{GHz} \mathrm{mm}$-wave to the system has the Q-factor is equal to 67.909 as shown in Figure 6(b). These two values of Q-factor are high enough for having a good quality of the output signal and have achieved the desired value of the Q-factor. BER for the downstream signal is zero which means the Q-factor has reached its maximum. Figure 7 portrays the eye diagram of the downstream signals. From this figure, it can be clearly seen that the opening eye is large and clear which means this signal is very good with little noise and distortion and it is easy to distinguish between logic 0 and logic $1[8,9]$.

\subsection{Upstream Design Analysis}

Before running the upstream design, seven iterations have to be set in order to operate the design. Seven iterations are needed because each upstream signal from any
Table 1. Downstream signal details with and without 40GHz mm-wave.

\begin{tabular}{ccc}
\hline & $\begin{array}{c}\text { Without 40-GHz } \\
\text { mm-wave }\end{array}$ & $\begin{array}{c}\text { With 40-GHz } \\
\text { mm-wave }\end{array}$ \\
\hline Max. Q-factor & 133.819 & 67.909 \\
Min. BER & 0 & 0 \\
Eye height & 0.008254238 & 0.00765657 \\
Threshold & 0.00200062 & 0.00347784 \\
Decision inst & 0.5 & 0.5 \\
\hline
\end{tabular}

individual user will go through seven time delays before reaching the upstream receiver. Table 2 shows the most important details of the upstream signal with and without the mm-wave. From this table, the BER values are larger than the BER values of the downstream signals; however, the Q-factor values are smaller than those of the downstream design as shown as in Figure 8.

This decrease in Q-factor and the increase in BER values lead us to have a signal with lower quality than the quality of the downstream signals. Even thought the upstream signal is a good signal, it is not as good as the downstream signal. The eye diagram of the upstream signals shows in Figure 9, that this signal has noise and distortion which are larger than those of the downstream signal. 


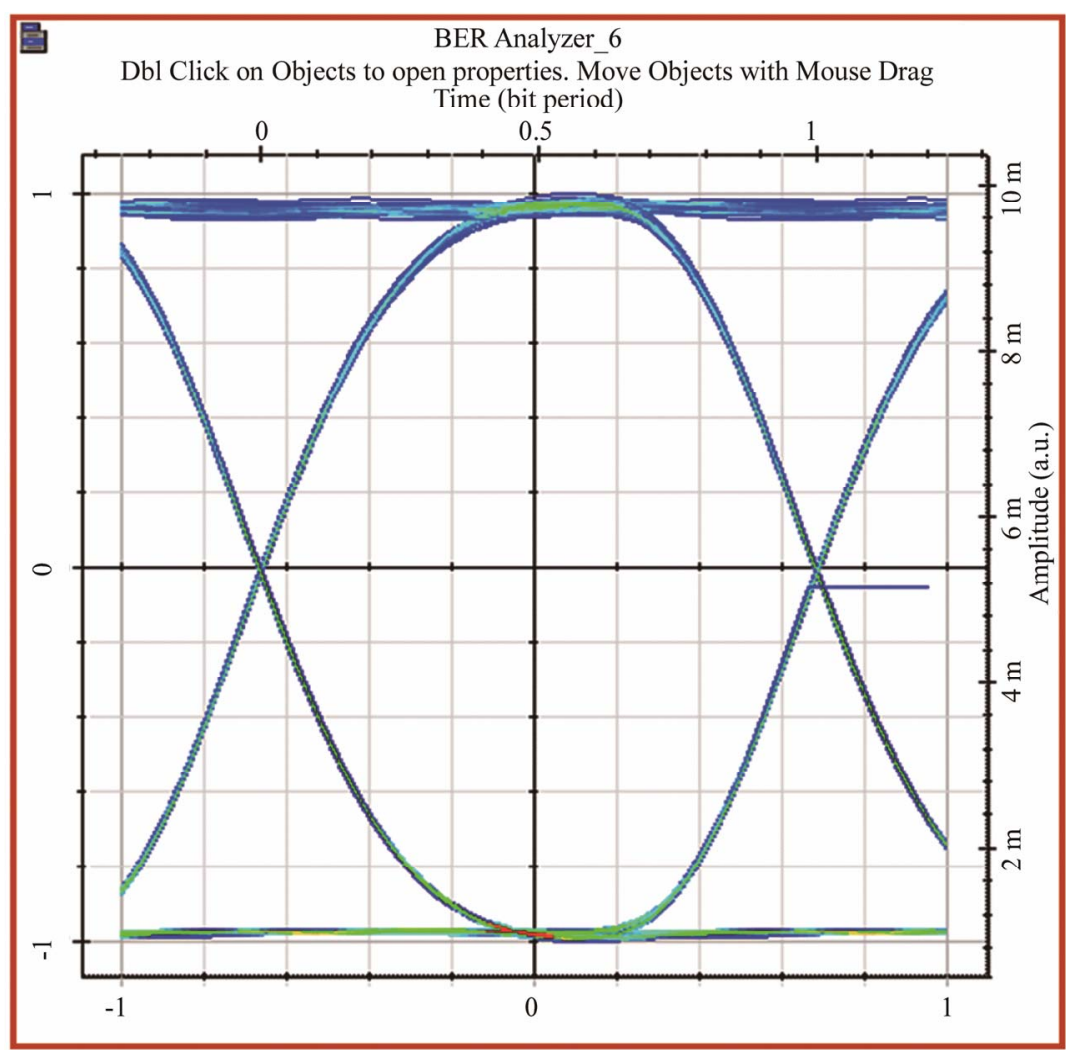

(a)

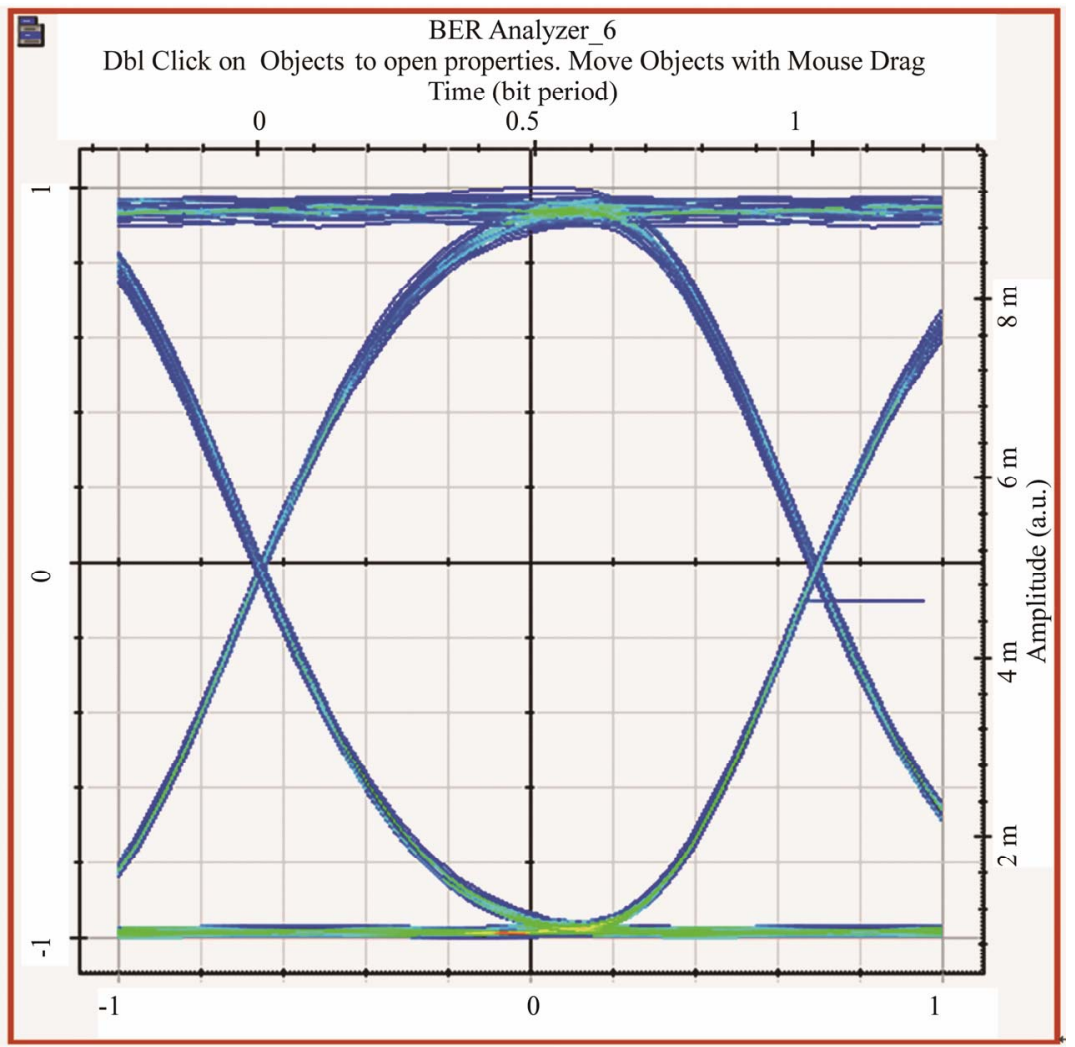

(b)

Figure 7. Eye diagram of the downstream signal with (b) and without (a) inserting 40-GHz mm-wave. 
Table 2. Upstream signal details with and without $\mathrm{Rf}$ generator.

\begin{tabular}{ccc}
\hline & Without Rf generator & With Rf generator \\
\hline Max. Q-factor & 8.09842 & 3.90774 \\
Min. BER & $1.6922 \mathrm{e}-016$ & $1.50805 \mathrm{e}-005$ \\
Eye height & 0.000345197 & $5.84756 \mathrm{e}-005$ \\
Threshold & 0.000318945 & 0.000103043 \\
Decision inst & 0.375 & 0.359375 \\
\hline
\end{tabular}

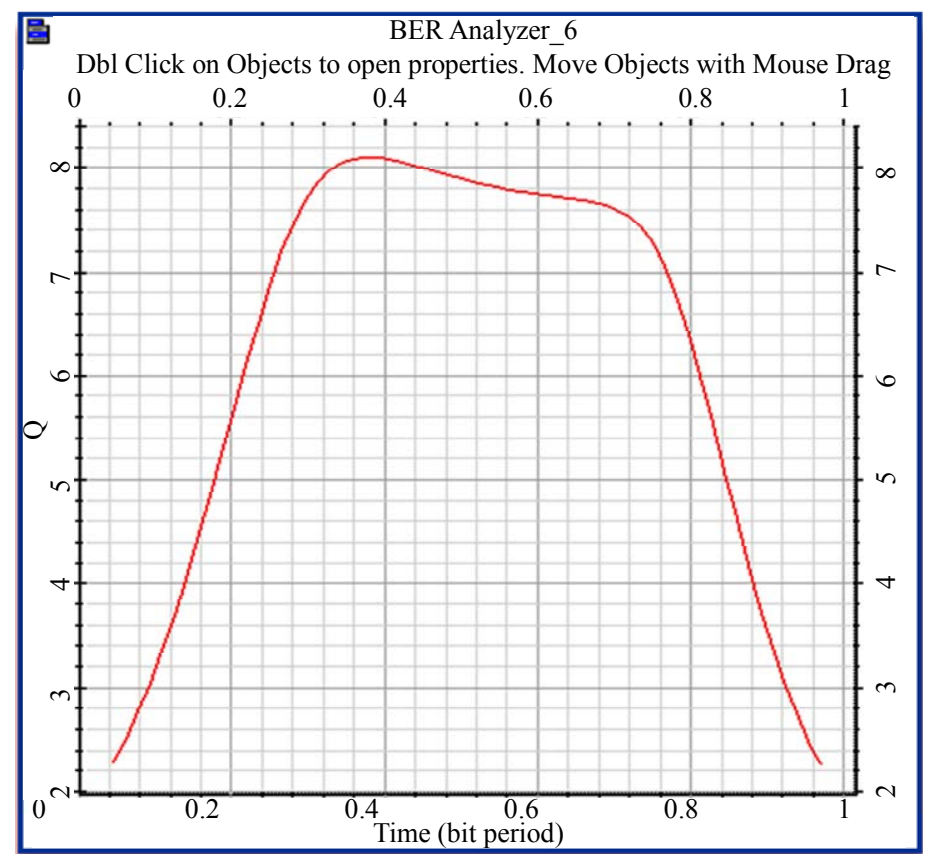

(a)

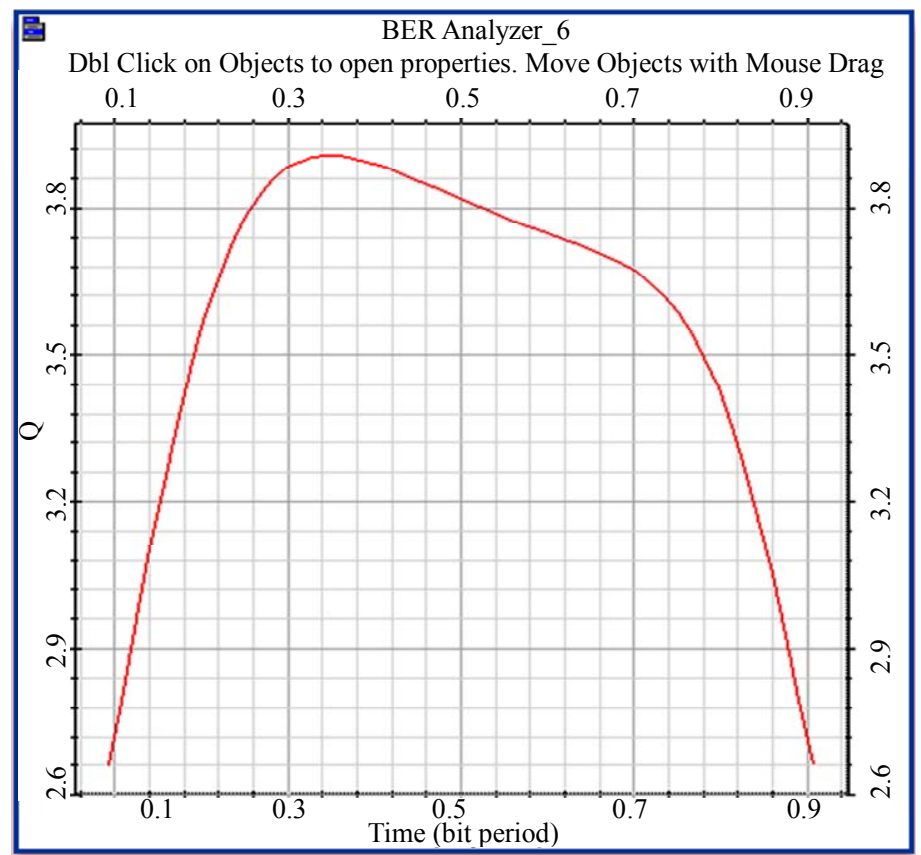

(b)

Figure 8. Q-factor vs. time for upstream signal with (b) and without (a) inserting 40-GHz mm-wave. 


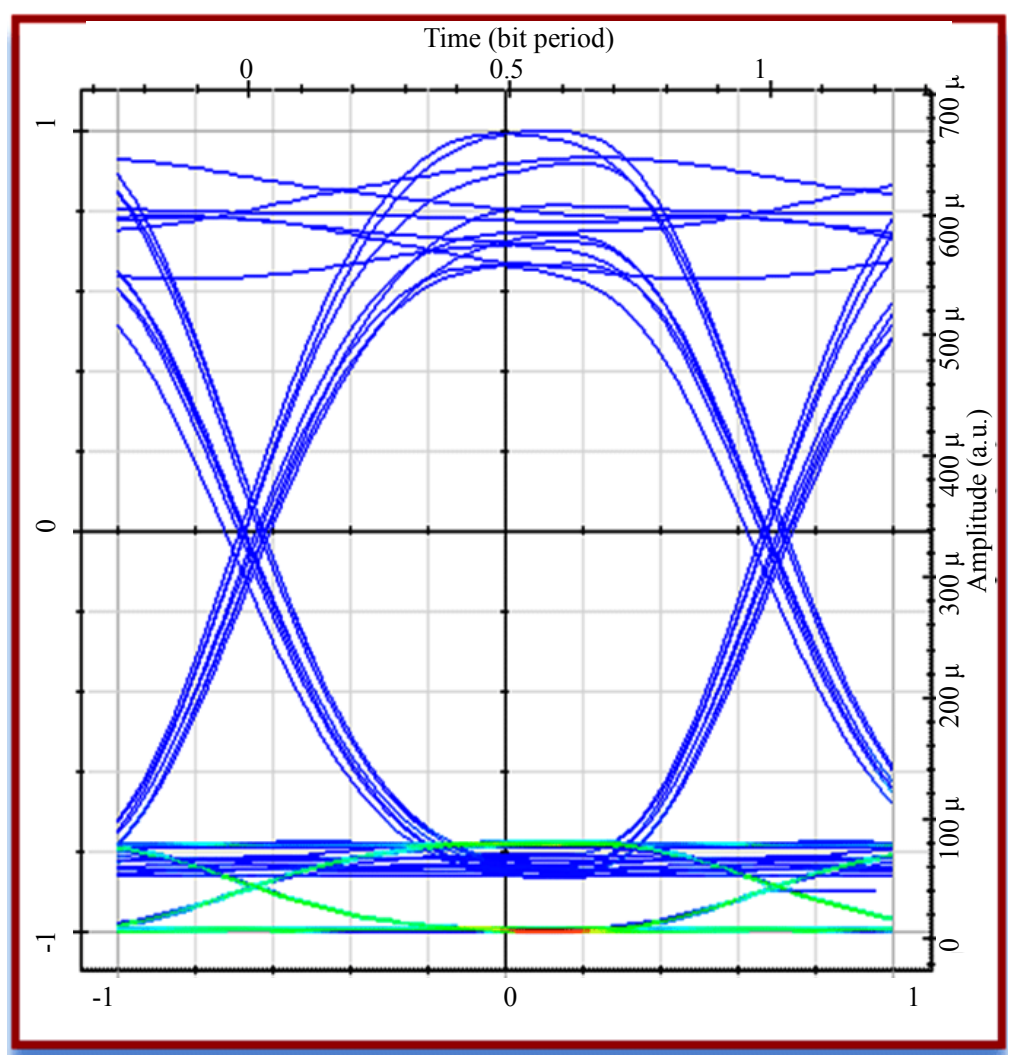

(a)

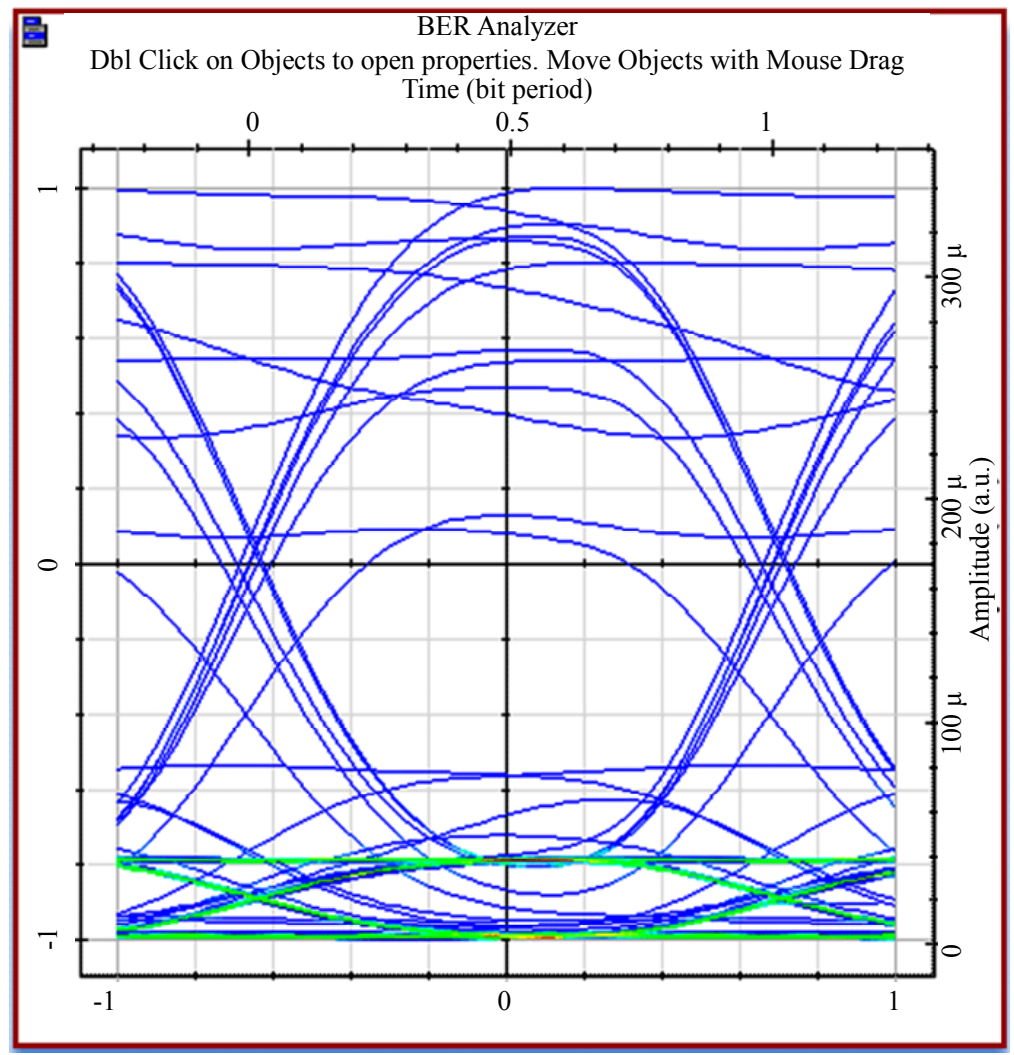

(b)

Figure 9. Eye diagram of the upstream signal with (b) and without (a) inserting 40-GHz mm-wave. 


\section{Conclusion}

The results showed that the BER of the signal was very low and the eye diagram showed the quality of the signal for the downstream was very high before adding the 40-GHz mm-wave; however, after adding the mm-wave to the system BER has increased and Q-factor has decreased as a result the quality of the signal was affected by $\mathrm{mm}$-wave. But for the upstream signals, as the eye diagram showed, the quality was good but not as high as the downstream signal because in the downstream a lot of equipment was used including amplifiers and filters. The qualities of the signals were decreased after adding the mm-wave; however, there are still acceptable. Building BPON system in general has showed high reliability. In general using PON has a lot of advantages which include allow costs, very low power consumption and flexibility in extending the network.

\section{REFERENCES}

[1] J. S. A. Pla, "Design of Passive Optical Network," Department of Telecommunications, Faculty of Electrical Engineering and Communication, Brno University of Technology, Brno, 2011.

[2] W. Stephen and T. Wang, "The Comsoc Guide to Passive Optical Networks Enhancing the Last Mile Access," John Wiley \& Sons, Chichester, 2012.

[3] R. Poboril, J. Latal, P. Koudelka, J. Vitasek, P. Siska, J.
Skapa and V. Vasinek, "A Conncept of a Hybrid WDM/ TDM Topology Using the Fabry Perot Laser in the Optiwave Simulation Environment," Advances in Electrical and Electronic Engineering, Vol. 9, No. 4, 2012, pp. $167-$ 178.

[4] Z. Jia, "Optical Millimeter-Wave Signal Generation, Transmission and Processing for Symmetric Super-Broadband Optical-Wireless Access Networks," Ph.D. Dissertation, Electrical Engineering, Georgia Tech, 2008.

[5] L.-J. Zhang, X.-J. Xin, B. Liu, Q. Zhang, Y.-J. Wang and C.-X. Yu, "Broadband Convergence of $40 \mathrm{GHz}-\mathrm{ROF}$ and $10-\mathrm{Gb} / \mathrm{s}$ WDM-PON Systems in the Duplex Access Network," Chinese Physics Letters, Vol. 27, No. 5, 2010, p. 054203. doi:10.1088/0256-307X/27/5/054203

[6] S. Rajalakshmi, A. Srivastava and A. Pandey, "RSOA Based 10 G WDM for Long Reach PON Using Manchester Coding for Re-Modulation," International Journal of Engineering Science and Technology, Vol. 4, No. 4, 2012, pp. 1767-1775.

[7] N. S. Afifi, M. Othman and Z. Tukiran, "Optimization for the Best Performance for Wavelength Division Multiplexed Passive Optical Network," Proceedings of Engineering Conference, 2008, pp. 1038-1043.

[8] G. Keiser, "Optical Fiber Communications," McGrawHill Education, New York, 2011.

[9] J. Zhang and N. Ansari, "Design of WDM PON with Tunable Lasers: The Upstream Scenario," Journal of Lightwave Technology, Vol. 28, No. 2, 2010, pp. 228236. 